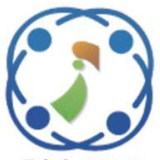

\title{
Fault Tolerant Control Based on Adaptive Fuzzy Global Fast Dynamic Terminal Sliding Mode for Actuators Failures in an Octorotor UAV
}

\author{
Abdelmoula Saggai $^{1} \quad$ Samir Zeghlache $^{1 *} \quad$ Loutfi Benyettou $^{2}$ \\ ${ }^{1}$ Laboratoire de Génie Electrique, Department of Electrical Engineering, Faculty of Technology, \\ University Mohamed Boudiaf of M'Sila, BP 166, Ichbilia 28000, Algeria \\ ${ }^{2}$ Laboratoire d'Analyse des Signaux et Systèmes, Department of Electrical Engineering, Faculty of Technology, \\ University Mohamed Boudiaf of M'Sila, BP 166, Ichbilia 28000, Algeria \\ * Corresponding author's Email: samir.zeghlache@univ-msila.dz
}

\begin{abstract}
This work presents a reliable fault-tolerant tracking control system (FTTCS) for actuator faults in a octorotor unmanned aerial vehicle. The proposed FTTCS is designed based on adaptive fuzzy global fast dynamic terminal sliding mode control (AFGFDTSMC) that guarantees the global asymptotic stability of a octorotor system. To mitigate the negative impacts of model uncertainties and enhance system robustness in faulty operation, an adaptive fuzzy system is incorporated into the global fast dynamic terminal sliding mode control (GFDTSMC) scheme for adaptively identifying the model uncertainties online and compensate the actuator faults effect. Lyapunov stability analysis proofs that the developed control method design maintains the stability in the closed loop dynamics of octorotor UAV in faulty operation. Simulation results show that the proposed (FTTCS) enables the octorotor to track the desired reference commands in the presence of actuator faults with satisfactory performance.
\end{abstract}

Keywords: Sliding mode control, Adaptive fuzzy logic systems, Non-linear control, Octorotor.

\section{Introduction}

For more than half a century Unmanned Aerial Vehicles (UAV) has been designed in the military field [1]. The objective was to replace the human being when the task to be accomplished became painful or repetitive and especially when it was a hostile environment where the pilot's safety was no longer assured.

Multi rotors helicopter modeling presents a challenge because this system is classified as nonlinear, underactuated, strongly coupled and has an unstable dynamic. In order to solve the trajectory tracking problems for this type of systems, some control strategies have been developed in the literature. Linear control approaches such as PID control can be used for attitude and altitude stabilization of a quadrotor UAV [2, 3]. Optimal linear control was also used for the control of multi rotors helicopter. In the works presented in [4-6], the Linear Quadratic Regulator (LQR) control technique was applied to control the linear model of quadrotor helicopter. $\mathrm{H} \infty \mathrm{o}$ methods are used in quadrotor helicopter control to synthesize controllers to obtain stabilization with best performance [7]. The development of linear control laws to control the motion of a multi rotors helicopter poses many problems because simplified linear models are generally far from the reality of the physical system. The complete dynamic model of a helicopter in fact generates uncertainties that constitute errors of the dynamics compared to the linear model, and, consequently, makes the elaboration of linear control laws very difficult. So the best solution is to synthesize nonlinear controls based on a complete modeling of the physical system. Nonlinear controller designs are generally based on the concept of closed-loop linearization [8,9] of the helicopter's non-linear model. The idea is to transform nonlinear dynamics into linear form using state feedback, with the input-state linearization corresponding to a full or partial linearization of the model. Other work, using 
the direct method of Lyapunov and taking into account the complete model of the quadrotor helicopter, was developed for trajectory tracking and stabilization at a fixed position [10-12]. The sliding mode control is characterized by robustness with respect to structural uncertainties and external disturbances for the control of drones [13]. However, the main disadvantage associated with this methodology is the appearance of vibrations (chattering phenomena) in the actuators that can damage the system. Better results have been obtained with the nonlinear backstepping control technique; the convergence of the internal states of the quadrotor has been guaranteed whatever the initial states [14]. This control technique was later reinforced with the addition of the integral action [15]. This approach has been validated to the quadrotor helicopter in various flight experiments. The control strategies proposed previously in the literature are generally based on the classical structure of quadrotor which leads to a deficiency of driving capacity especially in the presence of defects. A novel eight-rotor UAV model proposed in [16] has been used in this paper. The main advantages of this configuration are increased payload capacity, fault tolerance, stability in the wind and quietness efficiency. The FTC schemes enable a drone to continue flying with an acceptable capability despite the attendance of defects, over the past decades, researchers have classified FTC approaches into two types: passive fault-tolerant control (PFTC) and active fault-tolerant control (AFTC). PFTC uses robust control techniques to ensure system insensitivity to closed loop faults. It can maintain the stability of the system when the fault occurs. With PFTC, the system continues to operate with the same controller and system structure, usually; this technique is used in the case where the diagnosis of the fault is difficult to obtain. The PFTC does not require any fault detection and diagnosis system or controller reconfiguration. AFTC is based on online fault compensation and requires online fault information, therefore, this approach needs reconfiguration based on the information provided by the fault detection and identification (FDI) block [17]. This paper proposes an adaptive fuzzy global fast dynamic terminal sliding mode control (AFGFDTSMC) method for a class of unknown multiple-input-multiple-output (MIMO) nonlinear systems with bounded external and internal disturbances (octorotor helicopter with defective actuators) in order to compensate the fault effect after estimating uncertainties. Compared to recent work reported in the literature, the contributions of this paper are presented under different aspects such as: fault modeling, control strategy, type of multi rotors helicopter processed and type of fault:

- Unlike in [18], the adaptive FTC strategy, developed in this work, has been introduced in all steps of the global fast dynamic terminal sliding mode control (GFDTSMC) design, to obtain a better trajectory tracking in the presence of defects. Moreover, the developed control method deals with an octorotor helicopter in faulty operation, while in [18], a healthy classical quadrotor case is used.

- This work consists in proposing a new control strategy to improve the dynamic performance of the octorotor helicopter, especially in case of faulty operation.

- Unlike the passive FTC presented in [19], the proposed control structure is not bounded and can compensate the faults without information of their upper bounds, such as: actuator faults.

- Compared to the work of [20], the proposed FTC does not need an observer or internal model to compensate the fault effect.

- By using Lyapunov's method, the tracking errors are proven to be exponentially stable in contrast to the literature [21] where stability is not proven. In addition the proposed AFGFDTSMC controller can eliminate the chattering phenomenon which characterizes the sliding mode control.

This paper is organized as follows: In section 2, the dynamic model of octorotor helicopter in healthy condition is given. In section 3, a defective model of the octorotor helicopter is presented. The proposed FTC design is carried out in section 4. Simulation results are presented and analyzed in section 5 . Finally, the conclusion is given in section 6 .

\section{Dynamic modeling of the octorotor helicopter in healthy operation}

The octorotor as its name suggests is a helicopter with eight rotors, each rotor is composed of a motor, reducer and propeller. The configuration of the octorotor considered in this work is represented in Fig.1.

Unlike the quadrotor which only has four rotors, the octorotor has eight rotors equally spaced at $22.5^{\circ}$ and connected to the centre by arms [16]. To compute the dynamic model of the octorotor helicopter we will make the following assumptions [22]:

- The helicopter structure is rigid and symmetrical;

- The propellers are rigid;

- Thrust and drag are proportional to the square of the propellers speed;

- Ground effect is neglected;

- Each motor reacts immediately to the commanded rotor speed. 


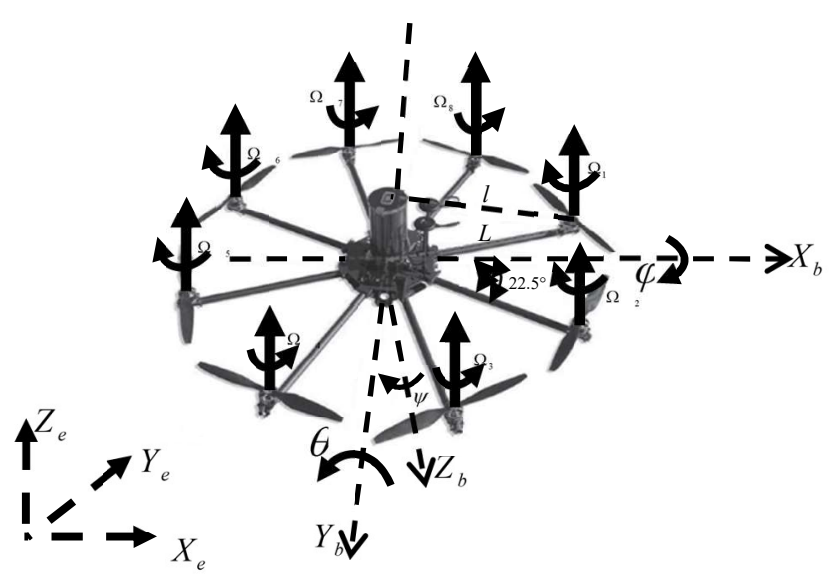

Figure. 1 Octorotor configuration [16]

Based on the above assumptions and using the Euler-Lagrange formulation, the dynamic model of the octorotor is given as the following form [16]:

$$
\left\{\begin{array}{l}
\ddot{\varphi}=\frac{1}{I_{x}}\left\{\dot{\theta} \dot{\psi}\left(I_{y}-I_{z}\right)-K_{1} \dot{\varphi}^{2}-J_{H} \vartheta \dot{\theta}+U_{2}\right\} \\
\ddot{\theta}=\frac{1}{I_{y}}\left\{\dot{\varphi} \dot{\psi}\left(I_{z}-I_{x}\right)-K_{2} \dot{\theta}^{2}-J_{H} \vartheta \dot{\varphi}+U_{3}\right\} \\
\ddot{\psi}=\frac{1}{I_{z}}\left\{\dot{\varphi} \dot{\theta}\left(I_{x}-I_{y}\right)-K_{3} \dot{\psi}^{2}+U_{4}\right\} \\
\ddot{x}=\frac{1}{m}\left\{(\cos \varphi \sin \theta \cos \psi+\sin \varphi \sin \psi) U_{1}-K_{4} \dot{x}\right\} \\
\ddot{y}=\frac{1}{m}\left\{(\cos \varphi \sin \theta \sin \psi-\sin \varphi \cos \psi) U_{1}-K_{5} \dot{y}\right\} \\
\ddot{z}=\frac{1}{m}\left\{(\cos \varphi \cos \theta) U_{1}-K_{6} \dot{z}\right\}-g
\end{array}\right.
$$

The variable $\vartheta$ in (1) is the overall residual propeller angular speed obtained by:

$\vartheta=-\Omega_{1}-\Omega_{2}+\Omega_{3}+\Omega_{4}-\Omega_{5}-\Omega_{6}+\Omega_{7}+\Omega_{8}$

Where $\Omega_{1}, \ldots, \Omega_{8}$ the angular speeds of the propeller, the other parameters are given in Table 1.

The mathematical formulation of the octorotor control inputs is obtained by [16]:
Table 1. Octorotor helicopter parameter definitions [16]

\begin{tabular}{|c|c|}
\hline Symbol & Parameter \\
\hline$m$ & mass \\
\hline$I_{\mathrm{x}}, I_{\mathrm{y}}$ and $I_{\mathrm{z}}$ & inertia on the $(x, y, z)$ axis \\
\hline$b$ & lift coefficient \\
\hline$d$ & force to moment scaling factor \\
\hline$J_{H}$ & arm and moment arm length $l=$ \\
\hline$L$ and $l$ & $\left.\frac{\pi}{8}\right)$ \\
\hline$K_{i}(i=1, \ldots, 6)$ & drag coefficients \\
\hline$g$ & acceleration of gravity \\
\hline$U_{1}$ & axis \\
\hline$U_{1}$ and $U_{3}$ & roll and pitch inputs \\
\hline$U_{4}$ & yawing moment \\
\hline
\end{tabular}

$$
\left[\begin{array}{l}
U_{1} \\
U_{2} \\
U_{3} \\
U_{4}
\end{array}\right]=\left[\begin{array}{cccccccc}
b & b & b & b & b & b & b & b \\
0 & 0 & -b l & -b l & 0 & 0 & b l & b l \\
b l & b l & 0 & 0 & -b l & -b l & 0 & 0 \\
-d & -d & d & d & -d & -d & d & d
\end{array}\right]\left[\begin{array}{l}
\Omega_{1}^{2} \\
\Omega_{2}^{2} \\
\Omega_{3}^{2} \\
\Omega_{4}^{2} \\
\Omega_{5}^{2} \\
\Omega_{6}^{2} \\
\Omega_{7}^{2} \\
\Omega_{8}^{2}
\end{array}\right]
$$

Where: $\Omega_{i}(i=1, \ldots 8)$ denotes the motors speeds.

\section{Main title dynamic modeling of the octorotor helicopter in faulty operation}

The dynamic model of the octorotor helicopter in defected case is given by:

$$
\left\{\begin{array}{l}
\ddot{\varphi}=\frac{1}{I_{x}}\left\{\dot{\theta} \dot{\psi}\left(I_{y}-I_{z}\right)-K_{1} \dot{\varphi}^{2}-J_{H} \vartheta \dot{\theta}+U_{2 f}\right\} \\
\ddot{\theta}=\frac{1}{I_{y}}\left\{\dot{\varphi} \dot{\psi}\left(I_{z}-I_{x}\right)-K_{2} \dot{\theta}^{2}-J_{H} \vartheta \dot{\varphi}+U_{3 f}\right\} \\
\ddot{\psi}=\frac{1}{I_{z}}\left\{\dot{\varphi} \dot{\theta}\left(I_{x}-I_{y}\right)-K_{3} \dot{\psi}^{2}+U_{4 f}\right\} \\
\ddot{x}=\frac{1}{m}\left\{(\cos \varphi \sin \theta \cos \psi+\sin \varphi \sin \psi) U_{1 f}-K_{4} \dot{x}\right\} \\
\ddot{y}=\frac{1}{m}\left\{(\cos \varphi \sin \theta \sin \psi-\sin \varphi \cos \psi) U_{1 f}-K_{5} \dot{y}\right\} \\
\ddot{z}=\frac{1}{m}\left\{(\cos \varphi \cos \theta) U_{1 f}-K_{6} \dot{z}\right\}-g
\end{array}\right.
$$


The fault in each rotor is modeled with a bias which multiplies to the forces in healthy operation obtained by:

$$
\Omega_{i f}=\left(1-\beta_{i}\right) \Omega_{i} ; \quad 0 \leq \beta_{i} \leq 1 \quad(i=1 \ldots 8)
$$

Where $\beta_{i}$ denotes the fault coefficient of each actuator, if $\beta_{i}=0$ alludes to healthy actuator and if $\beta_{i}=1$ reflect a completely damaged actuator.

$U_{1 f}, U_{2 f}, U_{3 f}$ and $U_{4 f}$ are the control inputs of the octorotor in faulty operation written by:

$$
\left[\begin{array}{l}
U_{1 f} \\
U_{2 f} \\
U_{3 f} \\
U_{4 f}
\end{array}\right]=\left[\begin{array}{cccccccc}
b & b & b & b & b & b & b & b \\
0 & 0 & -b l & -b l & 0 & 0 & b l & b l \\
b l & b l & 0 & 0 & -b l & -b l & 0 & 0 \\
-d & -d & d & d & -d & -d & d & d
\end{array}\right]\left[\begin{array}{l}
\left(1-\beta_{1}\right) \Omega_{1}^{2} \\
\left(1-\beta_{2}\right) \Omega_{2}^{2} \\
\left(1-\beta_{3}\right) \Omega_{3}^{2} \\
\left(1-\beta_{4}\right) \Omega_{4}^{2} \\
\left(1-\beta_{5}\right) \Omega_{5}^{2} \\
\left(1-\beta_{6}\right) \Omega_{6}^{2} \\
\left(1-\beta_{7}\right) \Omega_{7}^{2} \\
\left(1-\beta_{8}\right) \Omega_{8}^{2}
\end{array}\right]
$$

Introducing Eq. (6) into Eq. (4) yields:

$$
\left\{\begin{array}{l}
\ddot{\varphi}=\frac{1}{I_{x}}\left\{\dot{\theta} \dot{\psi}\left(I_{y}-I_{z}\right)-K_{1} \dot{\varphi}^{2}-J_{H} \bar{\Omega} \dot{\theta}+U_{2}\right\}+d_{\varphi} \\
\ddot{\theta}=\frac{1}{I_{y}}\left\{\dot{\varphi} \dot{\psi}\left(I_{z}-I_{x}\right)-K_{2} \dot{\theta}^{2}-J_{H} \bar{\Omega} \dot{\varphi}+U_{3}\right\}+d_{\theta} \\
\ddot{\psi}=\frac{1}{I_{z}}\left\{\dot{\varphi} \dot{\theta}\left(I_{x}-I_{y}\right)-K_{3} \dot{\psi}^{2}+U_{4}\right\}+d_{\psi} \\
\ddot{x}=\frac{1}{m}\left\{(\cos \varphi \sin \theta \cos \psi+\sin \varphi \sin \psi) U_{1}-K_{4} \dot{x}\right\}+d_{x} \\
\ddot{y}=\frac{1}{m}\left\{(\cos \varphi \sin \theta \sin \psi-\sin \varphi \cos \psi) U_{1}-K_{5} \dot{y}\right\}+d_{y} \\
\ddot{z}=\frac{1}{m}\left\{(\cos \varphi \cos \theta) U_{1}-K_{6} \dot{z}\right\}-g+d_{z}
\end{array}\right.
$$

The unknown resultant actuator faults are obtained by:

$$
\left\{\begin{aligned}
d_{\varphi}= & \frac{b l}{I_{x}}\left(\beta_{3} \Omega_{3}^{2}+\beta_{4} \Omega_{4}^{2}-\beta_{7} \Omega_{7}^{2}-\beta_{8} \Omega_{8}^{2}\right) \\
d_{\theta}= & \frac{b l}{I_{y}}\left(-\beta_{1} \Omega_{1}^{2}-\beta_{2} \Omega_{2}^{2}+\beta_{5} \Omega_{5}^{2}+\beta_{6} \Omega_{6}^{2}\right) \\
d_{\psi}= & \frac{d}{I_{z}}\left(\beta_{1} \Omega_{1}^{2}+\beta_{2} \Omega_{2}^{2}-\beta_{3} \Omega_{3}^{2}-\beta_{4} \Omega_{4}^{2}+\right. \\
& \left.\beta_{5} \Omega_{5}^{2}+\beta_{6} \Omega_{6}^{2}-\beta_{7} \Omega_{7}^{2}-\beta_{8} \Omega_{8}^{2}\right) \\
d_{x}= & \frac{\cos \varphi \sin \theta \cos \psi+\sin \varphi \sin \psi}{m}\left(-\beta_{1} \Omega_{1}^{2}-\beta_{2} \Omega_{2}^{2}-\right. \\
& \left.\beta_{3} \Omega_{3}^{2}-\beta_{4} \Omega_{4}^{2}-\beta_{5} \Omega_{5}^{2}-\beta_{6} \Omega_{6}^{2}-\beta_{7} \Omega_{7}^{2}-\beta_{8} \Omega_{8}^{2}\right) \\
d_{y}= & \frac{\cos \varphi \sin \theta \sin \psi-\sin \varphi \cos \psi}{m}\left(-\beta_{1} \Omega_{1}^{2}-\beta_{2} \Omega_{2}^{2}-\right. \\
& \left.\beta_{3} \Omega_{3}^{2}-\beta_{4} \Omega_{4}^{2}-\beta_{5} \Omega_{5}^{2}-\beta_{6} \Omega_{6}^{2}-\beta_{7} \Omega_{7}^{2}-\beta_{8} \Omega_{8}^{2}\right) \\
d_{z}= & \frac{\cos \varphi \cos \theta}{m}\left(-\beta_{1} \Omega_{1}^{2}-\beta_{2} \Omega_{2}^{2}-\beta_{3} \Omega_{3}^{2}-\beta_{4} \Omega_{4}^{2}-\right. \\
& \left.\beta_{5} \Omega_{5}^{2}-\beta_{6} \Omega_{6}^{2}-\beta_{7} \Omega_{7}^{2}-\beta_{8} \Omega_{8}^{2}\right)
\end{aligned}\right.
$$

\section{Assumption 2}

The actuator faults resultant related to the octorotor is bounded where:

$$
\left|d_{j}\right| \leq d_{j}^{+}, \quad j=\varphi, \theta, \psi, x, y, z \quad d_{j}^{+}>0
$$

\section{Design of FAGFDTSMC for octorotor helicopter in presence actuator faults}

The goal is to design an FTC based on FAGFDTSMC for an uncertain octorotor helicopter model in the presence of actuator faults to properly handle trajectory tracking. The role of fuzzy systems is to estimate the local nonlinearities of each subsystem by adaptive laws that respect the stability and convergence of the Lyapunov theory until the desired tracking performance is achieved. To design the proposed control, we operate with the defective octorotor dynamic model developed in (7), in the presence of actuator faults: 


$$
\left\{\begin{array}{l}
\ddot{\varphi}=\frac{1}{I_{x}} U_{2}+f_{1} \\
\ddot{\theta}=\frac{1}{I_{y}} U_{3}+f_{2} \\
\ddot{\psi}=\frac{1}{I_{z}} U_{4}+f_{3} \\
\ddot{x}=\frac{1}{m} U_{1}(\cos \varphi \sin \theta \cos \psi+\sin \varphi \sin \psi)+f_{4} \\
\ddot{y}=\frac{1}{m} U_{1}(\cos \varphi \sin \theta \sin \psi-\sin \varphi \cos \psi)+f_{5} \\
\ddot{z}=\frac{1}{m} U_{1}(\cos \varphi \cos \theta)+g+f_{6}
\end{array}\right.
$$

Where $f 1, \ldots, f 6$ are unknown functions given by:

$$
\left\{\begin{array}{l}
f_{1}=\frac{1}{I_{x}} \dot{\theta} \dot{\psi}\left(I_{y}-I_{z}\right)-\frac{K_{1}}{I_{x}} \dot{\varphi}^{2}-\frac{J_{H} \bar{\Omega}}{I_{x}} \dot{\theta}+d_{\varphi} \\
f_{2}=\frac{1}{I_{y}} \dot{\varphi} \dot{\psi}\left(I_{z}-I_{x}\right)-\frac{K_{2}}{I_{y}} \dot{\theta}^{2}+\frac{J_{H} \bar{\Omega}}{I_{y}} \dot{\varphi}+d_{\theta} \\
f_{3}=\frac{1}{I_{z}} \dot{\varphi} \dot{\theta}\left(I_{x}-I_{y}\right)-\frac{K_{3}}{I_{z}} \dot{\psi}^{2}+d_{\psi} \\
f_{4}=-\frac{K_{4}}{m} \dot{x}+d_{x} \\
f_{5}=-\frac{K_{5}}{m} \dot{y}+d_{y} \\
f_{6}=-\frac{K_{6}}{m} \dot{z}+d_{z}
\end{array}\right.
$$

The basic configuration of a fuzzy logic system consists of a fuzzifier, some fuzzy IF-THEN rules, a fuzzy inference engine and a defuzzifier [23], the general form of the $i^{t h}$ rule of the Takagi-SugenoKang (TSK) fuzzy logic system can be written as:

$R^{(i)}$ : if $x_{1} i s A_{1}^{i}$ and... and $x_{n}$ is $A_{n}^{i}$ then $\hat{f}$ is $y_{i}$

Where:

$x=\left[x_{1}, x_{2}, \ldots, x_{\mathrm{n}}\right]^{\mathrm{T}}$ are the inputs, $\hat{f}$ is the output, $A_{1}^{i}$, $A_{2}^{i}, \ldots$, and $A_{n}^{i}$ are the fuzzy sets and $y_{i}$ is the fuzzy singleton for the output in the $i^{t h}$ rule. The output of the fuzzy system is obtained by:

$f(x)=\frac{\sum_{i=1}^{m} y_{i}\left(\prod_{j=1}^{n} \mu_{A_{j}^{i}}\left(x_{j}\right)\right)}{\sum_{i=1}^{m}\left(\prod_{j=1}^{n} \mu_{A_{j}^{i}}\left(x_{j}\right)\right)}=\theta^{T} \psi(x)$
With $\theta^{T}=\left[y_{1}, y_{2}, \ldots, y_{m}\right]$ is the adjustable parameter vector, $m$ is the number of fuzzy rules, $\mu_{A j}^{i}$ is the degree of membership of $x_{j}$ to $\mu_{A}^{i}$, and $\quad \theta^{T}=\left[\psi_{l}\right.$, $\left.\psi_{2}, \ldots, \psi_{m}\right]$ is the fuzzy basis functions where:

$\psi_{i}(x)=\frac{\left(\prod_{j=1}^{n} \mu_{A_{j}^{i}}\left(x_{j}\right)\right)}{\sum_{i=1}^{m}\left(\prod_{j=1}^{n} \mu_{A_{j}^{i}}\left(x_{j}\right)\right)}$

The fuzzy inference system is utilized to approximate any nonlinearity $h_{i}\left(x_{i}\right)$ for $i=1, \ldots, 4$. This approximation function, which can be estimated on compact set $\vartheta_{x i}$, is obtained as:

$\hat{h}_{i}\left(\bar{x}_{i}\right)=\theta_{i}^{T} \psi_{i}\left(\bar{x}_{i}\right), i=1, \ldots, 4$

Where: $x_{i}$ is the input vector, $\theta_{i}$ is the adjusted vector parameters, and $\psi_{i}\left(x_{i}\right)$ is the fuzzy basis The optimal value of $\theta_{i}$ can be defined as:

$\theta_{i}^{*}=\underset{\theta_{i}}{\operatorname{Arg} \min }\left[\sup _{x_{i} \in \vartheta_{\bar{x}_{i}}}\left|h_{i}\left(\bar{x}_{i}\right)-\hat{h}_{i}\left(\bar{x}_{i}\right)\right|\right]$

Note that $\theta_{i}^{*}$, used only for demonstration purpose, is not required for controller synthesis [23-25].

The estimation error is expressed as:

$\tilde{\theta}_{i}=\theta_{i}^{*}-\theta_{i}$

The fuzzy approximation error is given as:

$\bar{\omega}_{i}\left(\bar{x}_{i}\right)=h_{i}\left(\bar{x}_{i}\right)-\theta_{i}^{* T} \psi_{i}\left(\bar{x}_{i}\right), i=1, \ldots, 4$

The compact set $\vartheta_{x i}$ is assumed large enough so that the input vector of the fuzzy systems remains in $\vartheta_{x i}$ under the closed loop control system. Consequently the fuzzy approximation error can be supposed to be bounded as presented in $[24,25]$ :

$\left|\bar{\omega}_{i}\left(\bar{x}_{i}\right)\right| \leq \omega_{i}, \quad \forall \bar{x}_{i} \in \vartheta_{\bar{x}_{i}}$

Where $\omega_{i}$ are unknown positive parameters.

The tracking errors and sliding surfaces are obtained by:

For z subsystem, the sliding surface is given by: 
$\tilde{z}(t)=z(t)-z^{*}, s_{z}=\dot{\tilde{z}}+c_{z} \tilde{z}$

The global fast dynamic terminal sliding surface is defined by [18]:

$S_{Z_{1}}=\dot{S}_{Z}+\lambda_{1} S_{Z}+\tau_{1} s_{Z}^{m_{1}^{\prime} / n_{1}^{\prime}}$

For $(y, \varphi)$ subsystems, the sliding surface is given by [26]:

$\tilde{y}(t)=y(t)-y^{*}, \tilde{\varphi}(t)=\varphi(t)-\varphi^{*}, s_{\varphi}=c_{1 \varphi} \dot{\tilde{y}}+$ $c_{2 \varphi} \tilde{y}+\dot{\tilde{\varphi}}+c_{3 \varphi} \tilde{\varphi}$

The global fast dynamic terminal sliding surface is defined by [18]:

$s_{\varphi_{1}}=\dot{s}_{\varphi}+\lambda_{2} S_{\varphi}+\tau_{2} s_{\varphi}^{m_{2}^{\prime} / n_{2}^{\prime}}$

For $(x, \theta)$ subsystems, the sliding surface is given by [26]:

$\tilde{x}(t)=x(t)-x^{*}, \tilde{\theta}(t)=\theta(t)-\theta^{*}, s_{\theta}=c_{1 \theta} \dot{\tilde{x}}+$

$c_{2 \theta} \tilde{x}+\dot{\tilde{\theta}}+c_{3 \theta} \tilde{\theta}$

The global fast dynamic terminal sliding surface is defined by [18]:

$s_{\theta_{1}}=\dot{s}_{\theta}+\lambda_{3} s_{\theta}+\tau_{3} s_{\theta}^{m^{\prime}{ }_{3} / n^{\prime}}$

For $\psi$ subsystem, the sliding surface is given by:

$\tilde{\psi}(t)=\psi(t)-\psi^{*}, s_{\psi}=\dot{\tilde{\psi}}+c_{\psi} \tilde{\psi}$,

The global fast dynamic terminal sliding surface is defined by [18]:

$s_{\psi_{1}}=\dot{s}_{\psi}+\lambda_{4} s_{\psi}+\tau_{4} s_{\psi}^{m^{\prime}{ }_{4} / n^{\prime}}$

Where $C_{1 \theta}, C_{2 \theta}, C_{3 \theta}, C_{l \varphi}, C_{1 \varphi}, C_{2 \varphi}, C_{3 \varphi}, C_{z}$ and $C_{\psi}$ are positive parameters, and we admit that:

$\varphi^{*}=\theta^{*}=0$
The adaptive fuzzy control laws presented in Eqs. (29)-(32) are written in the case where the dynamics of defective octorotor helicopter is uncertain:

$$
\begin{array}{r}
\dot{U}_{1}=\frac{m}{\cos \varphi \cos \theta}\left(-\theta_{1}^{T} \psi_{1}\left(\bar{x}_{1}\right)+\dddot{Z}^{*}+c_{Z} \ddot{Z}^{*}-\right. \\
\left(\lambda_{1}+\tau_{1} \frac{m_{1}^{\prime}}{n_{1}^{\prime}} s_{Z}\left(m^{\prime}{ }_{1}-n_{1}^{\prime}\right) / n^{\prime}{ }_{1}\right) \dot{s}_{Z}-k_{11} s_{Z_{1}}- \\
\left.k_{12} \tanh \left(\frac{s_{Z_{1}}}{\varepsilon_{z}}\right)\right)(29)
\end{array}
$$

$$
\begin{aligned}
& \dot{U}_{2}=I_{x}\left(-\theta_{2}^{T} \psi_{2}\left(\bar{x}_{2}\right)-\left(\lambda_{2}+\tau_{2} \frac{m_{2}^{\prime}}{n_{2}^{\prime}}\right.\right. \\
& \left.s_{\varphi}^{\left(m_{2}^{\prime}-n_{2}^{\prime}\right) / n_{2}^{\prime}}\right) \dot{s}_{\varphi}+ \\
& c_{1 \varphi} \dddot{y}^{*}+c_{2 \varphi} \ddot{y}^{*}+c_{3 \varphi} \ddot{\varphi}^{*}+\dddot{\varphi}^{*}-k_{21} s_{\varphi_{1}}- \\
& \left.k_{22} \tanh \left(\frac{s_{\varphi_{1}}}{\varepsilon_{\varphi}}\right)\right)(30)
\end{aligned}
$$

$$
\begin{array}{r}
\dot{U}_{3}=I_{y}\left(-\theta_{3}^{T} \psi_{3}\left(\bar{x}_{3}\right)\right. \\
-\left(\lambda_{3}+\tau_{3} \frac{m_{3}^{\prime}}{n_{3}^{\prime}} s_{\theta}\left(m^{\prime}{ }_{3}-n^{\prime}\right) / n_{3}^{\prime}\right) \dot{s}_{\theta}+ \\
c_{1 \theta} \dddot{x}^{*}+c_{2 \theta} \ddot{x}^{*}+c_{3 \theta} \ddot{\theta}^{*}+\dddot{\theta}^{*}-k_{31} s_{\theta_{1}}- \\
\left.k_{32} \tanh \left(\frac{s_{\theta_{1}}}{\varepsilon_{\theta}}\right)\right)(31)
\end{array}
$$

$$
\begin{gathered}
\dot{U}_{4}=\frac{1}{I_{z}}\left(-\theta_{4}^{T} \psi_{4}\left(\bar{x}_{4}\right)+\dddot{\psi}^{*}+c_{\psi} \ddot{\psi}^{*}-\right. \\
\left(\lambda_{4}+\tau_{4} \frac{m_{4}^{\prime}}{n_{4}^{\prime}} s_{\psi}\left(m^{\prime}{ }_{4}-n_{4}^{\prime}\right) / n^{\prime}{ }_{4}\right) \dot{s}_{\psi}-k_{41} s_{\psi_{1}}- \\
\left.k_{42} \tanh \left(\frac{s_{\psi_{1}}}{\varepsilon_{\psi}}\right)\right)
\end{gathered}
$$

With: $\varepsilon_{z}, \varepsilon_{\varphi}, \varepsilon_{\theta}$ and $\varepsilon_{z}$ are positive design parameters and the design parameters $k_{i 2}$ remain constants for $i=1, \ldots, 4$. The inputs of the fuzzy systems are given as:

$$
\begin{aligned}
& \bar{x}_{1}=[\tilde{z}, \dot{\tilde{z}}]^{T}, \quad \bar{x}_{2}=[\tilde{\varphi}, \dot{\tilde{\varphi}}]^{T}, \bar{x}_{3}=[\tilde{\theta}, \dot{\tilde{\theta}}]^{T}, \bar{x}_{4}= \\
& {[\tilde{\psi}, \dot{\tilde{\psi}}]^{T} .}
\end{aligned}
$$

In order to approximate the unknown fuzzy vectors $\left(\theta_{i}^{*}\right)$ and the unknown parameters $\left(k^{*}{ }_{i 2}\right)$ for $i=1, \ldots, 4$, we adopt the following adaptive laws [23, 27]:

$$
\left\{\begin{array}{l}
\dot{\theta}_{1}=-\sigma_{\theta 1} \gamma_{\theta 1} \theta_{1}+\gamma_{\theta 1} s_{z_{1}} \alpha_{1}\left(\bar{x}_{1}\right) \\
\dot{\theta}_{2}=-\sigma_{\theta 2} \gamma_{\theta 2} \theta_{2}+\gamma_{\theta 2} s_{\varphi_{1}} \alpha_{2}\left(\bar{x}_{2}\right) \\
\dot{\theta}_{3}=-\sigma_{\theta 3} \gamma_{\theta 3} \theta_{3}+\gamma_{\theta 3} s_{\theta_{1}} \alpha_{3}\left(\bar{x}_{3}\right) \\
\dot{\theta}_{4}=-\sigma_{\theta 4} \gamma_{\theta 4} \theta_{4}+\gamma_{\theta 4} s_{\psi_{1}} \alpha_{4}\left(\bar{x}_{4}\right)
\end{array}\right.
$$




$$
\left\{\begin{array}{l}
\dot{k}_{12}=-\sigma_{k 1} \gamma_{k 1} k_{12}+\gamma_{k 1} s_{z_{1}} \tanh \left(\frac{s_{z_{1}}}{\varepsilon_{z}}\right) \\
\dot{k}_{22}=-\sigma_{k 2} \gamma_{k 2} k_{22}+\gamma_{k 2} s_{\varphi_{1}} \tanh \left(\frac{s_{\varphi_{1}}}{\varepsilon_{\varphi}}\right) \\
\dot{k}_{32}=-\sigma_{k 3} \gamma_{k 3} k_{32}+\gamma_{k 3} s_{\theta_{1}} \tanh \left(\frac{s_{\theta_{1}}}{\varepsilon_{\theta}}\right) \\
\dot{k}_{42}=-\sigma_{k 4} \gamma_{k 4} k_{42}+\gamma_{k 4} s_{\psi_{1}} \tanh \left(\frac{s_{\psi_{1}}}{\varepsilon_{\psi}}\right)
\end{array}\right.
$$

Where: $\left(\sigma_{\theta i}, \gamma_{\theta i}, \gamma_{k i}, \sigma_{k i}\right)>0 \quad$ (for $\left.i=1, \ldots, 4\right)$; these parameters are design constants.

\section{Stability Analysis}

The proof stability is based on Lyapunov's theory. It is adopted by a feedback structure with two steps.

Step 1: From Eq. (10), the time derivative of $S_{z}$ is:

$\dot{S}_{z}=\frac{1}{m} U_{1}(\cos \varphi \cos \theta)-g+f_{6}-\ddot{z}^{*}+c_{z}\left(\dot{z}-\dot{z}^{*}\right)$

The time derivative of $S_{z l}$ is:

$$
\begin{gathered}
\dot{s}_{Z_{1}}=-c_{Z} \ddot{Z}^{*}-\dddot{Z}^{*}+\frac{1}{m} \dot{U}_{1}(\cos \varphi \cos \theta)+ \\
\left(\lambda_{1}+\tau_{1} \frac{m_{1}^{\prime}}{n_{1}^{\prime}} s_{Z}^{\left(m_{1}^{\prime}-n_{1}^{\prime}\right) / n_{1}^{\prime}}\right) \dot{s}_{Z}+h_{1}\left(\bar{x}_{1}\right)
\end{gathered}
$$

Where:

$$
h_{1}\left(\bar{x}_{1}\right)=c_{z}\left(\frac{1}{m} U_{1}(\cos \varphi \cos \theta)-g+f_{6}\right)+\dot{f}_{6}
$$

Defining the Lyapunov function for the z-position subsystem as:

$V_{1}=\frac{1}{2} s_{z 1}^{2}$

The time derivative of (36) is:

$$
\begin{array}{r}
\dot{V}_{1}=s_{z_{1}}\left(-c_{z} \ddot{Z}^{*}-\dddot{Z}^{*}+\frac{1}{m} \dot{U}_{1}(\cos \varphi \cos \theta)+\right. \\
\left.\left(\lambda_{1}+\tau_{1} \frac{m_{1}^{\prime}}{n_{1}^{\prime}} s_{Z}^{\left(m_{1}^{\prime}{ }^{\prime} n_{1}^{\prime}\right) / n_{1}^{\prime}}\right) \dot{s}_{z}+h_{1}\left(\bar{x}_{1}\right)\right)
\end{array}
$$

The following adaptive fuzzy system is developed to estimate the uncertain continuous function $h_{1}\left(x_{1}\right)$

$\widehat{h}_{1}\left(\bar{x}_{1}\right)=\theta_{1}^{T} \psi_{1}\left(\bar{x}_{1}\right)$

$$
\begin{aligned}
h_{1}\left(\bar{x}_{1}\right) & =\theta_{1}^{* T} \psi_{1}\left(\bar{x}_{1}\right)+\bar{\omega}_{1}\left(\bar{x}_{1}\right) \\
& =-\widetilde{\theta}_{1}^{T} \psi_{1}\left(\bar{x}_{1}\right)+\theta_{1}^{T} \psi_{1}\left(\bar{x}_{1}\right)+\bar{\omega}_{1}\left(\bar{x}_{1}\right)
\end{aligned}
$$

Where:

$\tilde{\theta}_{1}=\theta_{1}-\theta_{1}^{*}$

is the parameter error vector.

Introducing (40) into (39) yields:

$\dot{V}_{1}=-s_{z_{1}} \tilde{\theta}_{1}^{T} \psi_{1}\left(\bar{x}_{1}\right)+s_{z_{1}} \theta_{1}^{T} \psi_{1}\left(\bar{x}_{1}\right)+s_{z_{1}} \bar{\omega}_{1}\left(\bar{x}_{1}\right)+$

$s_{z_{1}} \frac{1}{m} \dot{U}_{1}(\cos \varphi \cos \theta)+\left(\lambda_{1}+\tau_{1} \frac{m_{1}^{\prime}}{n_{1}^{\prime}}\right.$

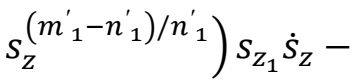

$$
s_{Z_{1}} c_{Z} \ddot{Z}^{*}-s_{z_{1}} \dddot{z}^{*}
$$

Where:

$\left|\bar{\omega}_{1}\left(\bar{x}_{1}\right)\right| \leq \omega_{1}$

$\omega_{1}$ : unknown constant

By selecting the control law obtained in Eq. (29) and using Eq. (43), we can make:

$\dot{V}_{1} \leq-s_{Z_{1}} \tilde{\theta}_{1}^{T} \alpha_{1}\left(\bar{x}_{1}\right)+k_{12}^{*}\left|s_{Z_{1}}\right|-k_{12}$

$s_{z_{1}} \tanh \left(\frac{s_{z}}{\varepsilon_{z}}\right)-k_{11} s_{z_{1}}^{2}$

Where: $k^{*}{ }_{12}=\omega_{1}$.

\section{Lemma 1}

The set $\left\{\varepsilon_{i}>0, x \in R\right\}$ satisfied the following inequality $[23,27]$ :

$$
\left\{\begin{array}{c}
0 \leq|x|-x \tanh \left(\frac{x}{\varepsilon_{i}}\right) \leq \varepsilon_{i}=\rho \varepsilon_{i} \quad\left[\varphi_{r}^{a b c}\right] \\
\rho=e^{-(1+\rho)} \simeq 0.2785
\end{array}\right.
$$

Using Eq. (45), Eq. (44) became:

$$
\begin{aligned}
& \dot{V}_{1} \leq-s_{z_{1}} \tilde{\theta}_{1}^{T} \alpha_{1}\left(\bar{x}_{1}\right)+k_{12}^{*} \bar{\varepsilon}_{z}-\tilde{k}_{12} s_{z_{1}} \tanh \left(\frac{s_{z_{1}}}{\varepsilon_{z}}\right)- \\
& k_{11} s_{z_{1}}^{2}
\end{aligned}
$$

Where:

$\tilde{k}_{12}=k_{12}-k_{12}^{*}$ and $\bar{\varepsilon}_{z}=0.2785 \varepsilon_{z}$

The augmented Lyapunov function is defined by: 
$V_{2}=V_{1}+\frac{1}{2 \gamma_{\theta_{1}}} \tilde{\theta}_{1}^{T} \tilde{\theta}_{1}+\frac{1}{2 \gamma_{k_{1}}} \tilde{k}_{12}^{2}$

The time derivative of Lyapunov function in Eq. (47) satisfied the following inequality:

$$
\begin{gathered}
\dot{V}_{2} \leq-s_{z_{1}} \tilde{\theta}_{1}^{T} \alpha_{1}\left(\bar{x}_{1}\right)+k_{12}^{*} \bar{\varepsilon}_{z}-\tilde{k}_{12} s_{z_{1}} \tanh \left(\frac{s_{z_{1}}}{\varepsilon_{z}}\right)- \\
k_{11} s_{z_{1}}^{2}+\frac{1}{2 \gamma_{\theta_{1}}} \tilde{\theta}_{1}^{T} \dot{\theta}_{1}+\frac{1}{2 \gamma_{k_{1}}} \tilde{k}_{12} \dot{k}_{12}
\end{gathered}
$$

By introducing Eqs. (34) and (35) in Eq. (48), then Eq. (48) will be bounded by the following formula:

$\dot{V}_{2} \leq k_{12}^{*} \bar{\varepsilon}_{z}-k_{11} s_{z_{1}}^{2}-\sigma_{\theta_{1}} \tilde{\theta}_{1}^{T} \theta_{1}-\sigma_{k_{1}} \tilde{k}_{12} k_{12}$

\section{Property:}

$$
\left\{\begin{array}{c}
-\tilde{\Theta}^{T} \Theta \leq-\frac{1}{2}\|\tilde{\Theta}\|^{2}+\frac{1}{2}\left\|\Theta^{*}\right\|^{2} \\
\tilde{\Theta}=\Theta-\Theta^{*} \in \mathfrak{R}^{n}
\end{array}\right.
$$

Where: $n$ is a positive integer number.

Utilizing (48), (47) is rewritten by:

$\dot{V}_{2} \leq-k_{11} s_{z_{1}}^{2}-\frac{\sigma_{\theta_{1}}}{2}\left\|\tilde{\theta}_{1}\right\|^{2}-\frac{\sigma_{k_{1}}}{2} \tilde{k}_{12}^{2}+\bar{\varepsilon}_{1}$

With:

$\bar{\varepsilon}_{1}=k_{12}^{*} \bar{\varepsilon}_{z}+\frac{\sigma_{\theta_{1}}}{2}\left\|\theta_{1}^{*}\right\|^{2}+\frac{\sigma_{k_{1}}}{2} k_{12}^{* 2}$

The stabilization of the filtered errors $s_{\varphi 1}, s_{\theta 1}$ and $s_{\psi 1}$ will be obtained in the second step.

Step 2: The objective of this step is to synthesis the control laws $\dot{U}_{2}, \dot{U}_{3}$ and $\dot{U}_{4}$.

In this step the Lyapunov function is defined by:

$V_{3}=V_{2}+\frac{1}{2} s_{\varphi_{1}}^{2}+\frac{1}{2} s_{\theta_{1}}^{2}+\frac{1}{2} s_{\psi_{1}}^{2}$

The time derivative of (53) checks the following inequality:

$$
\begin{gathered}
\dot{V}_{3} \leq- \\
-k_{11} s_{z_{1}}^{2}-\frac{\sigma_{\theta_{1}}}{2}\left\|\tilde{\theta}_{1}\right\|^{2}-\frac{\sigma_{k_{1}}}{2} \tilde{k}_{12}^{2}+\bar{\varepsilon}_{1}+s_{\varphi_{1}} \dot{s}_{\varphi_{1}} \\
+s_{\theta_{1}} \dot{s}_{\theta_{1}}+s_{\psi_{1}} \dot{s}_{\psi_{1}}
\end{gathered}
$$

The time derivative of filtered errors $s_{\varphi}, s_{\theta}$ and $s_{\psi}$ is computed using the equation (10):

$$
\left\{\begin{aligned}
\dot{s}_{\varphi}= & c_{1 \varphi}\left(\frac{1}{m} U_{1}(\cos \varphi \sin \theta \sin \psi-\sin \varphi \cos \psi)+\right. \\
& \left.f_{5}-\ddot{y}^{*}\right)+c_{2 \varphi}\left(\dot{y}-\dot{y}^{*}\right)+\frac{1}{I_{x}} U_{2}+ \\
& f_{1}-\ddot{\varphi}^{*}+c_{3 \varphi}\left(\dot{\varphi}-\dot{\varphi}^{*}\right) \\
\dot{s}_{\theta}= & c_{1 \theta}\left(\frac{1}{m} U_{1}(\cos \varphi \sin \theta \cos \psi+\sin \varphi \sin \psi)+\right. \\
& \left.f_{4}-\ddot{x}^{*}\right)+c_{2 \varphi}\left(\dot{x}-\dot{x}^{*}\right)+\frac{1}{I_{x}} U_{3}+ \\
& f_{2}-\ddot{\theta}^{*}+c_{3 \theta}\left(\dot{\theta}-\dot{\theta}^{*}\right) \\
\dot{s}_{\psi}= & \frac{1}{I_{z}} U_{4}+f_{3}-\ddot{\psi}^{*}+c_{\psi}\left(\dot{\psi}-\dot{\psi}^{*}\right)
\end{aligned}\right.
$$

The derivative of $s_{\varphi 1}, s_{\theta 1}$ and $s_{\psi 1}$ are given by:

$$
\left\{\begin{aligned}
\dot{s}_{\varphi_{1}}= & -c_{1 \varphi} \dddot{y}^{*}-c_{2 \varphi} \ddot{y}^{*}-c_{3 \varphi} \ddot{\varphi}^{*}-\dddot{\varphi}^{*}+ \\
& \frac{1}{I_{x}} \dot{U}_{2}+\left(\lambda_{2}+\tau_{2} \frac{m_{2}^{\prime}}{n_{2}^{\prime}} s_{\varphi}^{\left(m_{2}^{\prime}-n_{2}^{\prime}\right) / n_{2}^{\prime}}\right) \dot{s}_{\varphi}+h_{2}\left(\bar{x}_{2}\right) \\
\dot{s}_{\theta_{1}}= & -c_{1 \theta} \dddot{x}^{*}-c_{2 \theta} \ddot{x}^{*}-c_{3 \theta} \ddot{\theta}^{*}-\dddot{\theta}^{*}+ \\
& \frac{1}{I_{y}} \dot{U}_{3}+\left(\lambda_{3}+\tau_{3} \frac{m_{3}^{\prime}}{n_{3}^{\prime}} s_{\theta}^{\left.\left(m_{3}^{\prime}-n_{3}^{\prime}\right) / n_{3}^{\prime}\right)} \dot{s}_{\theta}+h_{3}\left(\bar{x}_{3}\right)\right. \\
\dot{s}_{\psi_{1}}= & -c_{z} \ddot{\psi}^{*}-\dddot{\psi}^{*}+\frac{1}{I_{z}} \dot{U}_{4}+ \\
& \left(\lambda_{4}+\tau_{4} \frac{m_{4}^{\prime}}{n_{4}^{\prime}} s_{\psi}^{\left(m_{4}^{\prime}-n_{4}^{\prime}\right) / n_{4}^{\prime}}\right) \dot{s}_{\psi}+h_{4}\left(\bar{x}_{4}\right)
\end{aligned}\right.
$$

Where: 


$$
\left\{\begin{aligned}
h_{2}\left(\bar{x}_{2}\right)= & c_{1 \varphi}\left(\frac{1}{m} \dot{U}_{1}(\cos \varphi \sin \theta \sin \psi-\sin \varphi \cos \psi)+\dot{f}_{5}\right)+ \\
& c_{2 \varphi}\left(\frac{1}{m} U_{1}(\cos \varphi \sin \theta \sin \psi-\sin \varphi \cos \psi)+f_{5}\right)+ \\
& \dot{f}_{1}+c_{3 \varphi}\left(\frac{1}{I_{x}} U_{2}+f_{1}\right) \\
h_{3}\left(\bar{x}_{3}\right)= & c_{1 \theta}\left(\frac{1}{m} \dot{U}_{1}(\cos \varphi \sin \theta \cos \psi+\sin \varphi \sin \psi)+\dot{f}_{4}\right)+ \\
& c_{2 \theta}\left(\frac{1}{m} U_{1}(\cos \varphi \sin \theta \cos \psi+\sin \varphi \sin \psi)+f_{4}\right)+ \\
& \dot{f}_{3}+c_{3 \theta}\left(\frac{1}{I_{y}} U_{3}+f_{2}\right) \\
h_{4}\left(\bar{x}_{4}\right)= & c_{\psi}\left(\frac{1}{I_{z}} U_{4}+f_{3}\right)+\dot{f}_{3}
\end{aligned}\right.
$$

By substituting Eq. (56) in Eq. (54), we obtain:

$$
\begin{aligned}
& \dot{V}_{3} \leq-k_{11} s_{z_{1}}^{2}+s_{\varphi_{1}}\left(-c_{1 \varphi} \dddot{y}^{*}-c_{2 \varphi} \ddot{y}^{*}-c_{3 \varphi} \ddot{\varphi}^{*}-\right. \\
& \dddot{\varphi}^{*}+\frac{1}{I_{x}} \dot{U}_{2}+ \\
& \left.\left(\lambda_{2}+\tau_{2} \frac{m_{2}^{\prime}}{n_{2}^{\prime}} S_{\varphi}^{\left(m_{2}^{\prime}-n_{2}^{\prime}\right) / n_{2}^{\prime}}\right) \dot{s}_{\varphi}+h_{2}\left(\bar{x}_{2}\right)\right)+ \\
& s_{\theta_{1}}\left(-c_{1 \theta} \dddot{x}^{*}-c_{2 \theta} \ddot{x}^{*}-c_{3 \theta} \ddot{\theta}^{*}-\dddot{\theta}^{*}+\frac{1}{I_{y}} \dot{U}_{3}+\right. \\
& \left.\left(\lambda_{3}+\tau_{3} \frac{m_{3}^{\prime}}{n_{3}^{\prime}} s_{\theta}^{\left(m_{3}^{\prime}-n_{3}^{\prime}\right) / n^{\prime}}\right) \dot{s}_{\theta}+h_{3}\left(\bar{x}_{3}\right)\right)+ \\
& s_{\psi_{1}}\left(-c_{z} \ddot{\psi}^{*}-\ddot{\psi}^{*}+\frac{1}{I_{z}} \dot{U}_{4}+\right. \\
& \left.\left(\lambda_{4}+\tau_{4} \frac{m_{4}^{\prime}}{n_{4}^{\prime}} S_{\psi}^{\left(m_{4}^{\prime}-n_{4}^{\prime}\right) / n^{\prime}}\right) \dot{S}_{\psi}+h_{4}\left(\bar{x}_{4}\right)\right)- \\
& \frac{\sigma_{\theta_{1}}}{2}\left\|\tilde{\theta}_{1}\right\|^{2}-\frac{\sigma_{k_{1}}}{2} \tilde{k}_{12}^{2}+\bar{\varepsilon}_{1}
\end{aligned}
$$

$h_{i}\left(x_{i}\right), i=2,3,4$ are unknown uncertainties functions, their estimation is performed by the following adaptive fuzzy system:

$$
\begin{aligned}
\hat{h}_{i}\left(\bar{x}_{i}\right) & =\theta_{i}^{T} \alpha_{i}\left(\bar{x}_{i}\right) \\
h_{i}\left(\bar{x}_{i}\right) & =\theta_{i}^{* T} \alpha_{i}\left(\bar{x}_{i}\right)+\bar{\omega}_{i}\left(\bar{x}_{i}\right) \\
& =-\tilde{\theta}_{i}^{T} \alpha_{i}\left(\bar{x}_{i}\right)+\theta_{i}^{T} \alpha_{i}\left(\bar{x}_{i}\right)+\bar{\omega}_{i}\left(\bar{x}_{i}\right)
\end{aligned}
$$

With: $x_{i}$ is pre-defined, $\omega_{i}\left(x_{i}\right)$ is the fuzzy approximation error with:

$\left|\bar{\omega}_{i}\left(\bar{x}_{i}\right)\right| \leq \omega_{i}$ $\omega_{i}$ is an unknown constant.

By choosing the adaptive control laws proposed in Eqs. (30)-(32) and the uncertainties functions $h_{i}\left(x_{i}\right)$ developed in Eq. (59), Eq. (58) is rewritten by:

$$
\begin{aligned}
& \dot{V}_{3} \leq-k_{11} s_{z_{1}}^{2}-\frac{\sigma_{\theta_{1}}}{2}\left\|\tilde{\theta}_{1}\right\|^{2}-\frac{\sigma_{k_{1}}}{2} \tilde{k}_{12}^{2}+\bar{\varepsilon}_{1}- \\
& s_{\varphi_{1}} \tilde{\theta}_{2}^{T} \alpha_{2}\left(\bar{x}_{2}\right)+k_{22}^{*}\left|s_{\varphi_{1}}\right|-k_{22} \\
& s_{\varphi_{1}} \tanh \left(\frac{s_{\varphi_{1}}}{\varepsilon_{\varphi}}\right)- \\
& k_{21} s_{\varphi_{1}}^{2}-s_{\theta_{1}} \tilde{\theta}_{3}^{T} \alpha_{3}\left(\bar{x}_{3}\right)+ \\
& k_{32}^{*}\left|s_{\theta_{1}}\right|-k_{32} s_{\theta_{1}} \tanh \left(\frac{s_{\theta_{1}}}{\varepsilon_{\theta}}\right)-k_{31} s_{\theta_{1}}^{2}- \\
& s_{\psi_{1}} \tilde{\theta}_{4}^{T} \alpha_{4}\left(\bar{x}_{4}\right)+k_{42}^{*}\left|s_{\psi_{1}}\right|- \\
& k_{42} s_{\psi_{1}} \tanh \left(\frac{s_{\psi_{1}}}{\varepsilon_{\psi}}\right)-k_{41} s_{\psi_{1}}^{2}
\end{aligned}
$$

Where $k_{22}^{*}=\omega_{2}, k_{32}^{*}=\omega_{3}$, and $k_{42}^{*}=\omega_{4}$. Using Eq. (45), the inequality (58) becomes:

$$
\begin{aligned}
& \dot{V}_{3} \leq-k_{11} s_{z_{1}}^{2}-\frac{\sigma_{\theta_{1}}}{2}\left\|\tilde{\theta}_{1}\right\|^{2}-\frac{\sigma_{k_{1}}}{2} \tilde{k}_{12}^{2}+\bar{\varepsilon}_{1}- \\
& s_{\varphi_{1}} \tilde{\theta}_{2}^{T} \alpha_{2}\left(\bar{x}_{2}\right)+ \\
& \quad k_{22}^{*} \bar{\varepsilon}_{\varphi}-\tilde{k}_{22} s_{\varphi_{1}} \tanh \left(\frac{s_{\varphi_{1}}}{\varepsilon_{\varphi}}\right)-k_{21} s_{\varphi_{1}}^{2}- \\
& s_{\theta_{1}} \tilde{\theta}_{3}^{T} \alpha_{3}\left(\bar{x}_{3}\right)+ \\
& k_{32}^{*} \bar{\varepsilon}_{\theta}-\tilde{k}_{32} s_{\theta_{1}} \tanh \left(\frac{s_{\theta_{1}}}{\varepsilon_{\theta}}\right)-k_{31} s_{\theta_{1}}^{2}- \\
& s_{\psi_{1}} \tilde{\theta}_{4}^{T} \alpha_{4}\left(\bar{x}_{4}\right)+ \\
& \quad k_{42}^{*} \bar{\varepsilon}_{\psi}-\tilde{k}_{42} s_{\psi_{1}} \tanh \left(\frac{s_{\psi_{1}}}{\varepsilon_{\psi}}\right)-k_{41} s_{\psi_{1}}^{2}
\end{aligned}
$$

Where:

$$
\begin{aligned}
& \tilde{k}_{22}=k_{22}-k_{22}^{*}, \tilde{k}_{32}=k_{32}-k_{32}^{*}, \tilde{k}_{42}=k_{42}- \\
& k_{42}^{*}, \bar{\varepsilon}_{\varphi}=0.2785 \varepsilon_{\varphi}, \bar{\varepsilon}_{\theta}=0.2785 \varepsilon_{\theta} \text { and } \bar{\varepsilon}_{\psi}= \\
& 0.2785 \varepsilon_{\psi}
\end{aligned}
$$

The augmented Lyapunov function is defined by:

$$
\begin{aligned}
V_{4}= & V_{3}+\frac{1}{2 \gamma_{\theta_{2}}} \tilde{\theta}_{2}^{T} \tilde{\theta}_{2}+\frac{1}{2 \gamma_{k_{2}}} \tilde{k}_{22}^{2}+\frac{1}{2 \gamma_{\theta_{3}}} \tilde{\theta}_{3}^{T} \tilde{\theta}_{3}+ \\
& \frac{1}{2 \gamma_{k_{3}}} \tilde{k}_{32}^{2}+\frac{1}{2 \gamma_{\theta_{4}}} \tilde{\theta}_{4}^{T} \tilde{\theta}_{4}+\frac{1}{2 \gamma_{k_{4}}} \tilde{k}_{42}^{2}
\end{aligned}
$$

The time derivation of Eq. (61) is given by:

$$
\begin{gathered}
\dot{V}_{4} \leq-k_{11} s_{z_{1}}^{2}-\frac{\sigma_{\theta_{1}}}{2}\left\|\tilde{\theta}_{1}\right\|^{2}-\frac{\sigma_{k_{1}}}{2} \tilde{k}_{12}^{2}+\bar{\varepsilon}_{1} \\
-s_{\varphi_{1}} \tilde{\theta}_{2}^{T} \alpha_{2}\left(\bar{x}_{2}\right)+ \\
k_{22}^{*} \bar{\varepsilon}_{\varphi}-\tilde{k}_{22} s_{\varphi_{1}} \tanh \left(\frac{s_{\varphi_{1}}}{\varepsilon_{\varphi}}\right)-k_{21} s_{\varphi_{1}}^{2}-
\end{gathered}
$$$$
s_{\theta_{1}} \tilde{\theta}_{3}^{T} \alpha_{3}\left(\bar{x}_{3}\right)+
$$ 


$$
\begin{aligned}
& k_{32}^{*} \bar{\varepsilon}_{\theta}-\tilde{k}_{32} s_{\theta_{1}} \tanh \left(\frac{s_{\theta_{1}}}{\varepsilon_{\theta}}\right)-k_{31} s_{\theta_{1}}^{2}- \\
& s_{\psi_{1}} \tilde{\theta}_{4}^{T} \alpha_{4}\left(\bar{x}_{4}\right)+ \\
& k_{42}^{*} \bar{\varepsilon}_{\psi}-\tilde{k}_{42} s_{\psi_{1}} \tanh \left(\frac{s_{\psi_{1}}}{\varepsilon_{\psi}}\right)-k_{41} s_{\psi_{1}}^{2}+ \\
& \frac{1}{2 \gamma_{\theta_{2}}} \tilde{\theta}_{2}^{T} \dot{\theta}_{2}+ \\
& \frac{1}{2 \gamma_{k_{2}}} \tilde{k}_{22} \dot{k}_{22}+\frac{1}{2 \gamma_{\theta_{3}}} \tilde{\theta}_{3}^{T} \dot{\theta}_{3}+ \\
& \frac{1}{2 \gamma_{k_{3}}} \tilde{k}_{32} \dot{k}_{32}+\frac{1}{2 \gamma_{\theta_{4}}} \tilde{\theta}_{4}^{T} \dot{\theta}_{4}+\frac{1}{2 \gamma_{k_{4}}} \tilde{k}_{42} \dot{k}_{42}
\end{aligned}
$$

By exploiting Eq. (50), yields:

$$
\begin{aligned}
& \dot{V}_{4} \leq-k_{11} s_{z_{1}}^{2}-\frac{\sigma_{\theta_{1}}}{2}\left\|\tilde{\theta}_{1}\right\|^{2}-\frac{\sigma_{k_{1}}}{2} \tilde{k}_{12}^{2}-k_{21} s_{\varphi_{1}}^{2}- \\
& \frac{\sigma_{\theta_{2}}}{2}\left\|\tilde{\theta}_{2}\right\|^{2}- \\
& \frac{\sigma_{k_{2}}}{2} \tilde{k}_{22}^{2}-k_{31} s_{\theta_{1}}^{2}-\frac{\sigma_{\theta_{3}}}{2}\left\|\tilde{\theta}_{3}\right\|^{2}-\frac{\sigma_{k_{3}}}{2} \tilde{k}_{32}^{2}-k_{41} \\
& s_{\psi_{1}}^{2}- \\
& \frac{\sigma_{\theta_{4}}}{2}\left\|\tilde{\theta}_{4}\right\|^{2}-\frac{\sigma_{k_{4}}}{2} \tilde{k}_{42}^{2}+\bar{\varepsilon}_{1}+\bar{\varepsilon}_{2}+\bar{\varepsilon}_{3}+\bar{\varepsilon}_{4}
\end{aligned}
$$

Where:

$\bar{\varepsilon}_{2}=k_{22}^{*} \bar{\varepsilon}_{\varphi}+\frac{\sigma_{\theta_{2}}}{2}\left\|\theta_{2}^{*}\right\|^{2}+\frac{\sigma_{k_{2}}}{2} k_{22}^{* 2}, \bar{\varepsilon}_{3}=k_{32}^{*} \bar{\varepsilon}_{\varphi}+$ $\frac{\sigma_{\theta_{3}}}{2}\left\|\theta_{3}^{*}\right\|^{2}+\frac{\sigma_{k_{3}}}{2} k_{32}^{* 2}$,

$\bar{\varepsilon}_{4}=k_{42}^{*} \bar{\varepsilon}_{z}+\frac{\sigma_{\theta_{4}}}{2}\left\|\theta_{4}^{*}\right\|^{2}+\frac{\sigma_{k_{4}}}{2} k_{42}^{* 2}$

Eq. (66) Can be simplified by:

$\dot{V}_{4} \leq-\eta V_{4}+\mu$

With:

$\mu=\bar{\varepsilon}_{1}+\bar{\varepsilon}_{2}+\bar{\varepsilon}_{3}+\bar{\varepsilon}_{4} \quad, \quad \eta_{k}=\min \left\{\sigma_{k_{1}} \gamma_{k_{1}}, \sigma_{k_{2}}\right.$

$\left.\gamma_{k_{2}}, \sigma_{k_{3}} \gamma_{k_{3}}, \sigma_{k_{4}} \gamma_{k_{4}}\right\}$

And:

$$
\begin{gathered}
\eta=\min \left\{2 k_{11}, 2 k_{21}, 2 k_{31}, 2 k_{41}, \sigma_{\theta_{1}} \gamma_{\theta_{1}},\right. \\
\left.\sigma_{\theta_{2}} \gamma_{\theta_{2}}, \sigma_{\theta_{3}} \gamma_{\theta_{3}}, \sigma_{\theta_{4}} \gamma_{\theta_{4}}, \eta_{k}\right\}
\end{gathered}
$$

The multiplication of Eq. (65) by the exponential term, $e^{\eta t}$ we get $[23,27]$ :

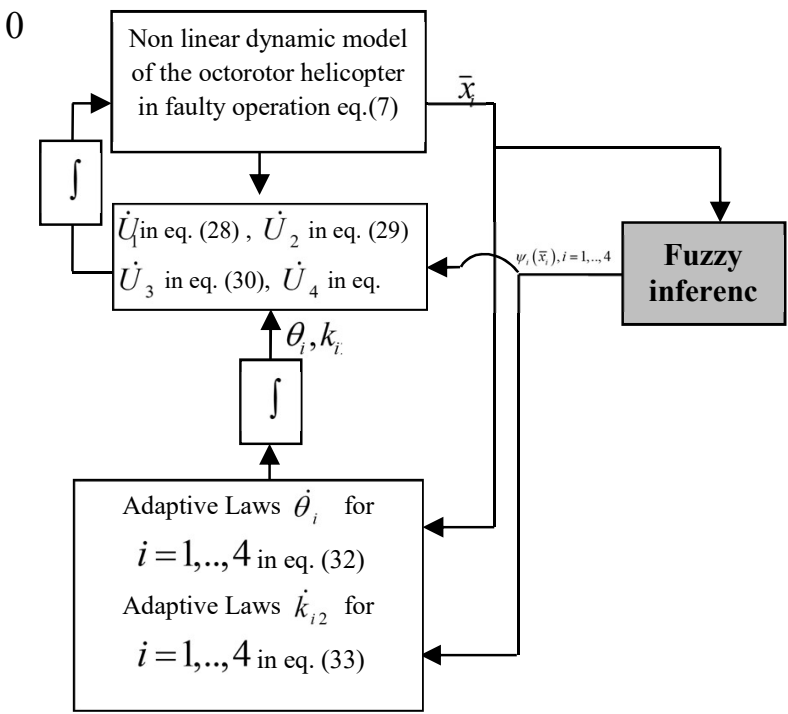

Figure. 2 organizational chart of the developed AFGFDTSMC system

$\frac{d}{d t}\left(V_{4} e^{\eta t}\right) \leq \mu e^{\eta t}$

The integration of (67) over $[0, t]$ yields:

$0 \leq V_{4} \leq \frac{\mu}{\eta}+\left(V_{4}(0)-\frac{\mu}{\eta}\right) e^{-\eta t}$

Where: $\eta$ is selected depending to the design parameters and $\mu$ is a randomly selected parameter. In accordance with [23, 27], the Lyapunov function in Eq. (62) is bounded also reflect the exponential convergence to an adjustable residual set for tracking and parameter estimation errors, which means that all signals in closed-loop are bounded. Fig. 2 presents the global block diagram of the proposed FTC.

The organizational chart of the developed AFGFDTSMC system is shown in Fig. 2.

\section{Simulation results}

To prove the efficacy and the capability for the path following problem of the octorotor helicopter, the developed FTC has been simulated. The physical parameters of octorotor helicopter are obtained by [16]: $m=1.3 \mathrm{~kg}, L=0.23 \mathrm{~m}$, the nominal values of $I_{x}$, $I_{y}$ and $I_{z}$ are $\left(I_{x}, I_{y}, I_{z}\right)=\operatorname{diag}\left(1.5 \times 10^{-3}, 1.5 \times 10^{-3}\right.$, $\left.2.6 \times 10^{-3}\right) \quad \mathrm{Kg} / \mathrm{m}^{2}, \quad b=3.13 \times 10^{-5} \mathrm{~N} / \mathrm{s}^{2}, \quad d=7.5 \times 10^{-7}$ $\mathrm{Nm} / \mathrm{s}^{2}, \quad J_{H}=6 \times 10^{-5} \mathrm{Kg} / \mathrm{m}^{2}, \quad k_{1}=k_{2}=k_{3}=10^{-3} \mathrm{~N} / \mathrm{m} / \mathrm{s}$, $k_{4}=k_{5}=k_{6}=1.2 \times 10^{-3} \mathrm{~N} / \mathrm{rad} / \mathrm{s}$.

The vectors:

$$
\begin{aligned}
& \bar{x}_{1}=[\tilde{z}, \dot{\tilde{z}}]^{T}, \bar{x}_{2}=[\tilde{\varphi}, \dot{\tilde{\varphi}}]^{T}, \bar{x}_{3}=[\tilde{\theta}, \dot{\tilde{\theta}}]^{T}, \bar{x}_{4}= \\
& {[\tilde{\psi}, \dot{\tilde{\psi}}]^{T}}
\end{aligned}
$$



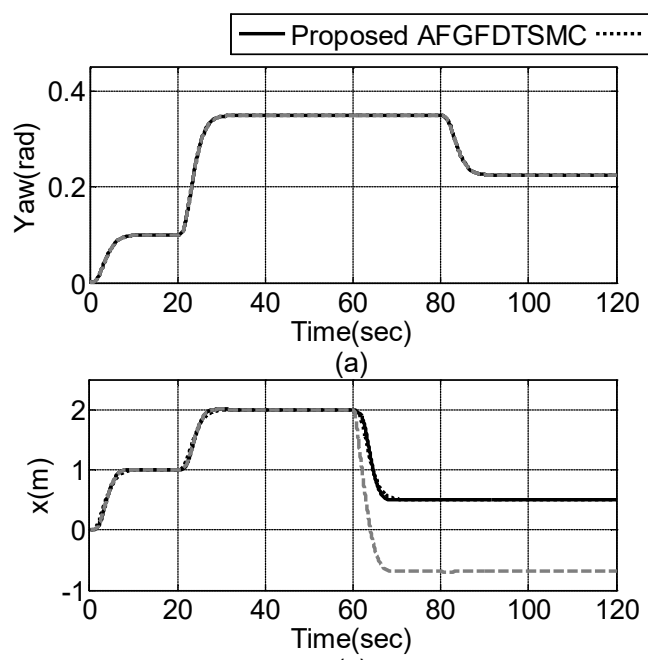

(c)
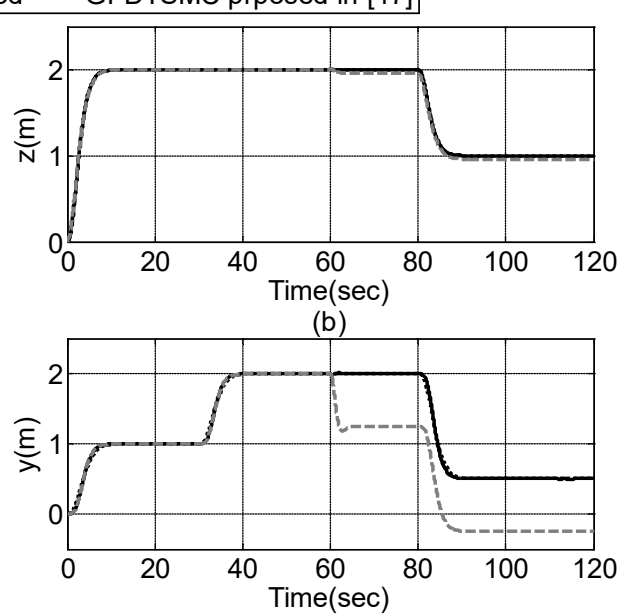

(d)

Figure. 3 Trajectory of the output variables (Rotor 2,4,6,8 fault and rotor 1,3 failure)

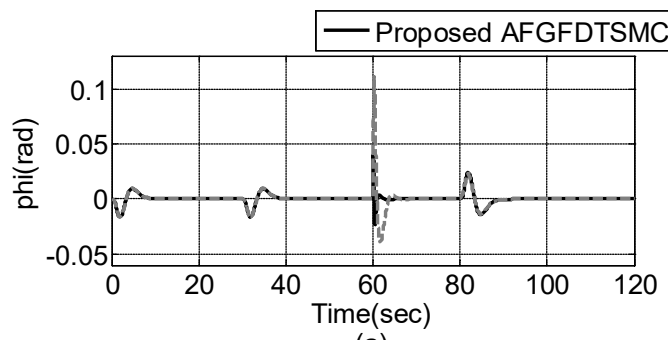

(a)

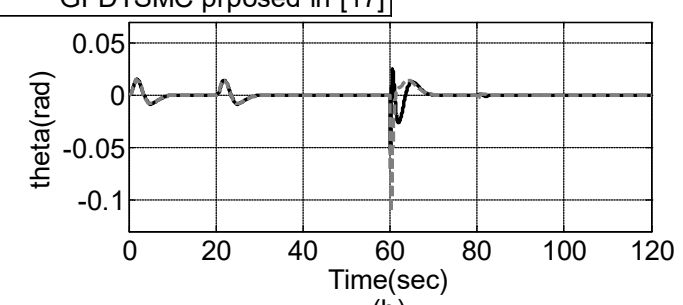

(b)

Figure. 4 Trajectories of roll and pitch angles (Rotor 2,4,6,8 fault and rotor 1,3 failure)

represent the inputs of the fuzzy inference systems $\theta_{\mathrm{i}}^{\mathrm{T}} \alpha\left(x_{i}\right)$ for $i=1, \ldots, 4$. For each variable we define seven Gaussian membership functions uniformly distributed on the intervals $[-1,1]$. Note that all the design adaptive parameters of the control laws are selected to reach a satisfactory transient control performance considering the requirement of stability and all variable states of the octorotor helicopter are supposed measurable. Flight tests in faulty condition have been carried out to demonstrate the performance of the proposed control.

\subsection{Rotor $2,4,6,8$ fault and rotor 1,3 failure}

Figs. 3 and 4 show the simulation results where more severe fault/failure scenario where rotors $2,4,6$ and 8 are only $50 \%$ effective from $40 \mathrm{sec}$ onwards, and then rotor 1 and 3 fail totally at $60 \mathrm{sec}$. Fig. 5 shows the evolution of rotor speed. The global trajectory effectuated by the octorotor helicopter in 3D is given in Fig. 6. According to Figs. 3, 4, and 5, it can be seen, the proposed control strategy can track the reference trajectory accurately despite actuators defects. In addition, it is obviously that the proposed AFGFDTSMC can give small following error and good tracking performance compared to GFDTSMC proposed in [18].

\section{Conclusion}

In this paper, the robust adaptive controller that is based on adaptive fuzzy global fast dynamic terminal sliding mode system has been investigated via its application in tracking control of octorotor helicopter. With this controller, the control law ensures the convergence of tracking errors in presence of actuator faults and parametric variations. This law incorporates adaptive parameters to compensate the faults effect. The obtained simulation results show that proposed controller keeps the tracking errors in an acceptable interval in the attendance of actuator defects and parametric variations. In addition, the comparative study performed with the control strategy proposed in [18] has shown the effectiveness of the proposed control approach in faulty operation. In the future work, the experimental implementation of the proposed control scheme will be addressed.

\section{Conflicts of Interest}

Declare conflicts of interest or state "The authors 


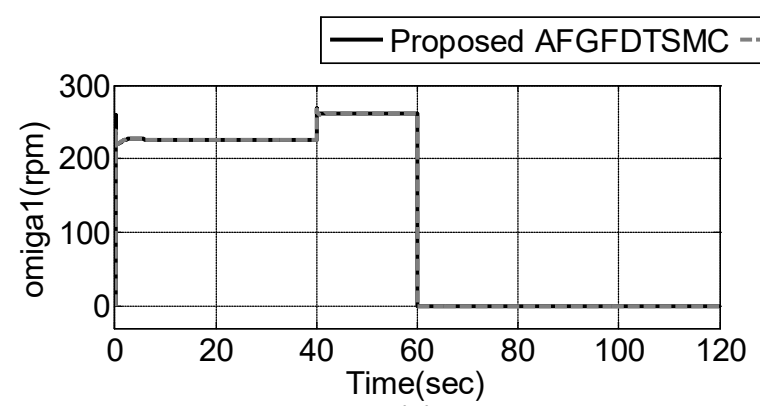

(a)

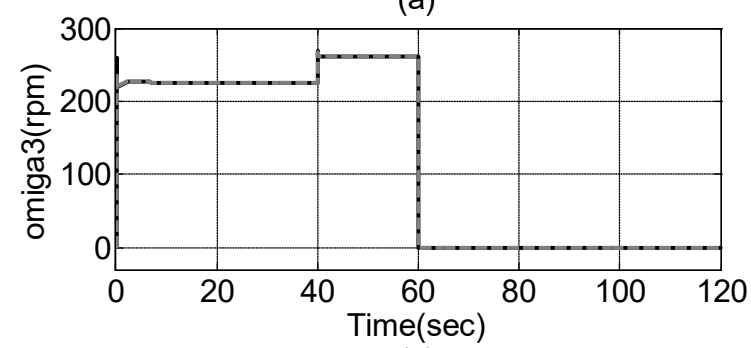

(c)

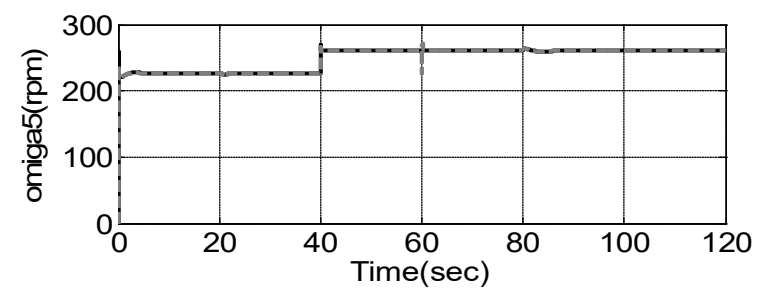

(e)

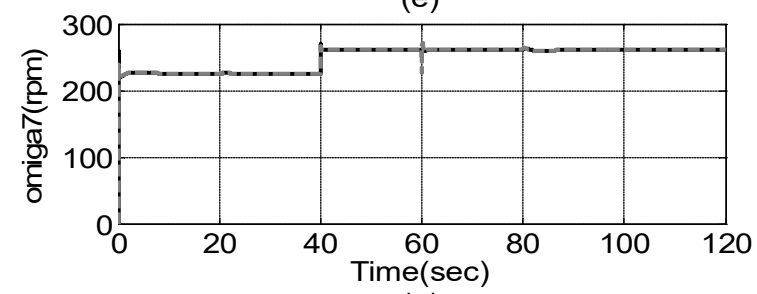

(g)

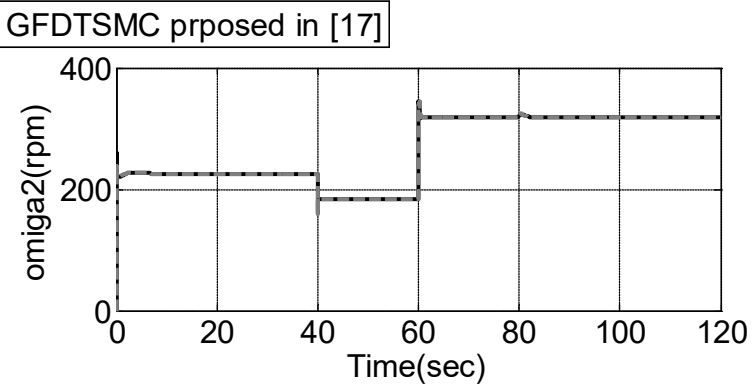

(b)

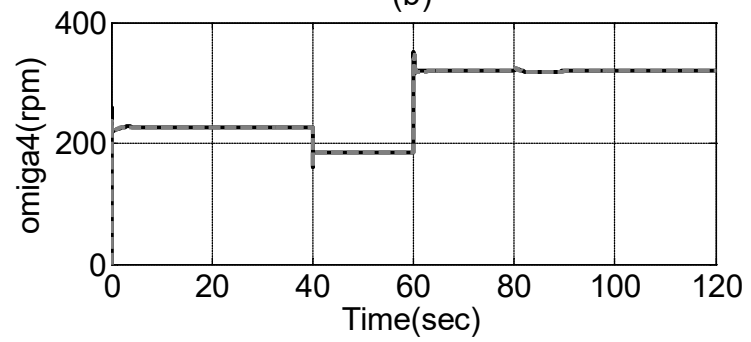

(d)

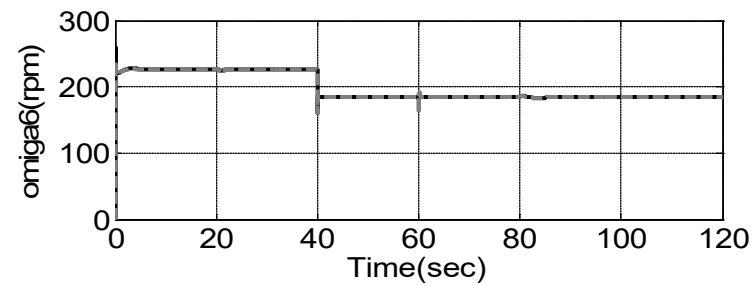

(f)

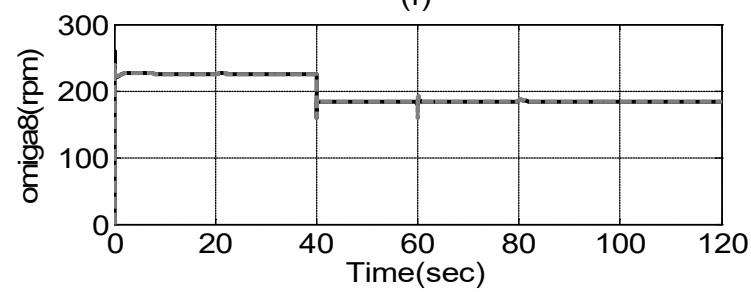

(h)

Figure. 5 Rotor speed (Rotor 2,4,6,8 fault and rotor 1,3 failure)

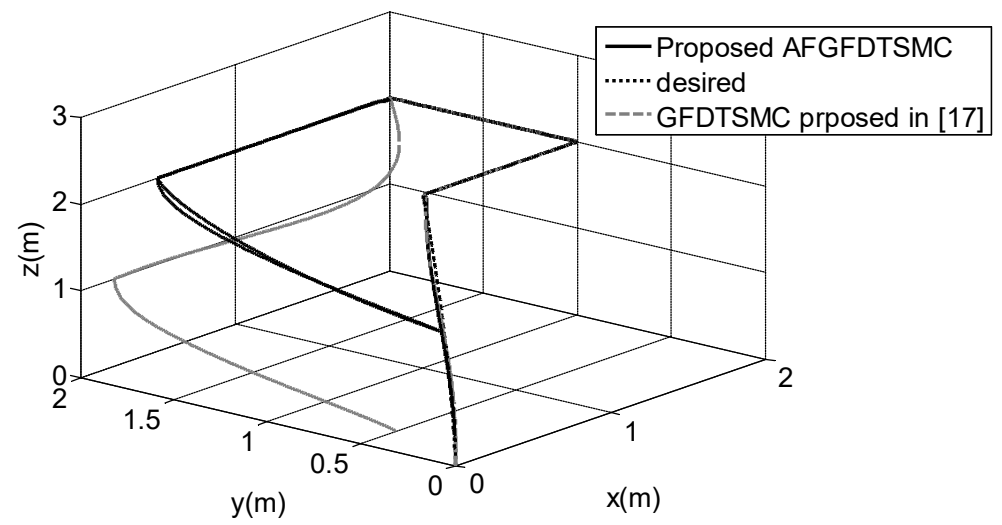

Figure. 6 System position in the 3D space (Rotor 2,4,6,8 fault and rotor 1,3 failure)

declare no conflict of interest." Authors must identify and declare any personal circumstances or interest that may be perceived as inappropriately influencing the representation or interpretation of reported research results.

\section{Author Contributions}

For research articles with several authors, a short paragraph specifying their individual contributions must be provided. The following statements should 
be used as follows: "Conceptualization, Abdelmoula Saggai; methodology, Loutfi Benyettou; software, Samir Zeghlache; formal analysis, Loutfi Benyettou; investigation, Abdelmoula Saggai; resources, Loutfi Benyettou; writing - writing - review and editing, Samir Zeghlache; visualization, Loutfi Benyettou; supervision, Samir Zeghlache.

\section{References}

[1] J. C. Yeon, A. R. Nyoman, and Y. S. Soo, "Cellular Communication-Based Autonomous UAV Navigation with Obstacle Avoidance for Unknown Indoor Environments", International Journal of Intelligent Engineering and Systems, Vol. 14, No. 2, pp. 344-352, 2021.

[2] J. Qingsong, L. Jia, and Z. Yunxi, "Analysis and design the controller for quadrotors based on PID control method", In: Proc. of the IEEE International Conference on Chinese Association of Automation, Changchun, China, pp. 88-92, 2018

[3] M. Abdel-Razzak , N. Hassan, and B. François, "Emergency Control of AR Drone Quadrotor UAV Suffering a Total Loss of One Rotor", IEEE/ASME Transactions on Mechatronics., Vol. 22, No. 2, pp. 961-971, 2017.

[4] B. S. Anjali and A. Vivek, J. L. "Nandagopal Simulation and Analysis of Integral LQR Controller for Inner Control Loop Design of a Fixed Wing Micro Aerial Vehicle (MAV)", Procedia Technology. Vol.25, pp. 76-83, 2016.

[5] A. Ramin, F. Rasul, and A. K. Mohammad, "Updating LQR Control for Full Dynamic of a Quadrotor", In: Proc. of the IEEE International Conference on Robotics and Mechatronics, Vienna Austria, pp. 279-285, 2017.

[6] L. Changlong, P. Jian, and C. Yufang, "PID and LQR trajectory tracking control for an unmanned quadrotor helicopter", In: Proc. of the IEEE International Conference on Chinese Control Conference, Chengdu, China, pp. 10845-10850, 2016.

[7] P. O. Juan , I. M. Luis, and J. R. Manuel, "Nonlinear Robust H-Infinity PID Controller for the Multivariable System Quadrotor", IEEE Latin America Transactions, Vol. 14, No. 3, pp. 1176-1183, 2016.

[8] T. Bailing, M. Yuxin, and A. Q. Zong, "Continuous Finite-Time Output Feedback control Scheme and Its Application in Quadrotor UAVs", IEEE Access., Vol. 6, pp. 19807-19813, 2018.

[9] E. C. Dong and E. Yongsoon, "Global Chartwise Feedback Linearization of the
Quadcopter With a Thrust Positivity Preserving Dynamic Extension", IEEE Transactions on Automatic Control, Vol. 62, No. 9, pp. 47474752, 2017.

[10] W. Yihao, L. Chuanjiang, and S. Yanchao, "Backstepping approach for controlling a quadrotor using Barrier Lyapunov Functions", In: Proc. of the 36th IEEE International Conference of Chinese Control Conference, Dalian, China, 2017.

[11] S. Ali and N. M. Muhammad, "Lyapunov-based nonlinear controller for quadrotor position and attitude tracking with GA optimization", In: Proc. of the IEEE International Conference on Industrial Electronics and Applications Conference, United States, pp. 342-347, 2016.

[12] X. Zhiwei, N. Xiaohong, W. Haibo, and C. Yinsheng, "Robust guaranteed cost tracking control of quadrotor UAV with uncertainties", ISA Transaction. Vol. 69, pp.157-165, 2017.

[13] C. MJutarut, T. Vittaya, and N. Arjin, "Modified Adaptive Sliding Mode Control for Trajectory Tracking of Mini-drone Quadcopter Unmanned Aerial Vehicle", International Journal of Intelligent Engineering and Systems, Vol. 13, No. 5, pp. 145-158, 2020.

[14] J. Tao, L. Defu, and S. Tao, "Finite-Time Backstepping Control for Quadrotors With Disturbances and Input Constraints", IEEE Access, Vol. 6, pp. 62037-62049, 2018.

[15] J. Zhenyue , Y. Jianqiao, M. Yuesong, C. Yongbo, S. Yuanchuan, and A. Xiaolin, "Integral backstepping sliding mode control for quadrotor helicopter under external uncertain disturbances", Aerospace Science and Technology, Vol. 68, pp.299-307,2017.

[16] A Halim and E. Christopher, "Sliding mode fault-tolerant control of an octorotor using linear parameter varying-based schemes", IET Control Theory Application, Vol. 9, No. 4, pp. 618-636, 2015.

[17] Y. Zhang, S. Tang, and J. Guo, "Adaptive-gain fast super-twisting sliding mode fault tolerant control for a reusable launch vehicle in reentry phase", ISA transactions, Vol. 71, No. 2, pp. 380-390, 2017.

[18] X. Jing-Jing and Z. Guo-Bao, "Global fast dynamic terminal sliding mode control for a quadrotor UAV", ISA Transactions, Vol. 66, pp. 233-240, 2017.

[19] W. Ban and Z. Youmin, "An Adaptive FaultTolerant Sliding Mode Control Allocation Scheme for Multirotor Helicopter Subject to Simultaneous Actuator Faults", IEEE 
Transactions on Industrial Electronics, Vol. 65, pp. 4227-4236, 2018.

[20] M. Hemza, B. Omar, B. Djamel, T. Mohamed, and B. Mohamed, "Sliding mode based fault detection, reconstruction and fault tolerant control scheme for motor systems", ISA Transactions, Vol. 57, pp. 340-351, 2015.

[21] K. Erdal and M. Reinaldo, "Type-2 Fuzzy Logic Trajectory Tracking Control of Quadrotor VTOL Aircraft With Elliptic Membership Functions", IEEE/ASME Transactions on Mechatronics, Vol. 22, No. 1, pp. 339-348, 2017.

[22] A. Marks, J. Whidborne, and I. Yamamoto, "Control allocation for fault tolerant control of a VTOL octorotor", In: Proc. of International Conference on Control (UKACC), Cardiff, UK, pp. 357-362, 2012.

[23] N. Bounar, A. Boulkroune, F. Boudjema, M. M'Saad, and M. Farza, "Adaptive fuzzy vector control for a doubly-fed induction motor", Neuro computing, Vol. 15, No. 2, pp. 756-769, 2015.

[24] A. Boulkroune and M. M'saad, "A fuzzy adaptive variable-structure control schemefor uncertain chaotic MIMO systems with sector nonlinearities and dead zones", Expert System Application, Vol. 38, pp. 14744-14750, 2011.

[25] A. Boulkroune and M. M'saad, "On the design of observer-based fuzzy adaptivecontroller for nonlinear systems with unknown control gain sign", Fuzzy SetsSystem, Vol. 201, pp. 71-85. 2012.

[26] Z. En-Hui, X. Jing-Jing and L. Ji-Liang, "Second order sliding mode control for a Quadrotor UAV", ISA Transaction, Vol. 53, No. 4, pp. 1350-1356, 2014.

[27] F. Yacef, O. Bouhali, and M. Hamerlain, N. Rizoug, "Observer-based adaptive fuzzy backstepping tracking control of quadrotor unmanned aerial vehicle powered by Li-ion battery", Journal of Intelligent \& Robotic Systems, Vol. 84, pp. 179-197, 2016. 